\title{
LIMIT DISTRIBUTIONS OF MANY-PARTICLE SPECTRA AND $q$-DEFORMED GAUSSIAN VARIABLES
}

\author{
PIOTR ŚNIADY \\ Institute of Mathematics, University of Wrockaw \\ Pl. Grunwaldzki 2/4, 50-384 Wrockaw, Poland \\ E-mail: Piotr.Sniady@math.uni.wroc.pl
}

\begin{abstract}
We find the limit distributions for a spectrum of a system of $n$ particles governed by a $k$-body interaction. The hamiltonian of this system is modelled by a Gaussian random matrix.

We show that the limit distribution is a $q$-deformed Gaussian distribution with the deformation parameter $q$ depending on the fraction $k / \sqrt{n}$. The family of $q$-deformed Gaussian distributions include the Gaussian distribution and the semicircular law; therefore our result is a generalization of the results of Wigner [Wig1, Wig2], Mon and French [MF].
\end{abstract}

\section{Introduction}

1.1. The $k$-body interactions. We say that the interaction of $n$ particles is a $k$-body interaction if it can be treated as a sum of independent interactions, each taking place in a group of $k$ particles (the groups of course need not be disjoint). The integer $k$ is called the rank of the interaction.

From basic principles of physics we would expect that the fundamental interactions should take place only in pairs of particles; by taking into account higher order interactions between carriers of interaction we see that also $k$-body interactions are possible if $k$ is a small integer.

Perhaps even better examples of a $k$-body interaction we obtain by considering models of complex physical systems which were simplified by disregarding some components. In such models effective hamiltonians contain also $k$-body interactions $(k>2)$ in order to preserve the effects connected to the disregarded components.

Nevertheless we would expect that $k$, the rank of the interaction, should be relatively small.

2000 Mathematics Subject Classification: 81V35, 81V70, 15A52.

The paper is in final form and no version of it will be published elsewhere. 


\subsection{Random matrix models for complex quantum systems}

1.2.1. Wigner's model. The first and the simplest random matrix model for complex quantum systems such as atomic nuclei was proposed by Wigner [Wig1]. In this model a hamiltonian of the system is represented by a large hermitian matrix $\left(a_{i j}\right)_{1 \leq i, j \leq N}$ which entries are complex Gaussian random variables. This model can be heuristically justified as follows: in an sufficiently complex physical system the matrix elements of the hamiltonian should be very complicated as well and therefore can be regarded as random.

In the Wigner's model the matrix element between any two physical states can take nonzero values, what for quantum systems consisting of $n$ parts means that the system is governed by an $n$-body interaction, what in the light of the preceding discussion is physically doubtful.

1.2.2. Random matrix model for $k$-body interaction. The following more realistic model was proposed [BF1, BF2, FW1, FW2, WF] in which a $k$-body interaction hamiltonian of $n$ particles is modeled by a random matrix.

We consider a quantum system of $n$ distinguishable particles; the Hilbert space $\mathcal{H}$ of the system is a tensor product of the Hilbert spaces $\mathcal{H}_{i}(1 \leq i \leq n)$ corresponding to particles. In fact as "particles" we can take also quantum statistical objects such as collective excitations, holes, etc.

We assume that the rank of the effective interaction in our system is equal to $k$ and therefore the hamiltonian $H$ of the system is a sum of hamiltonians $H_{A}$ of the $k$-particle subsystems,

$$
H=\sum_{A} H_{A}
$$

The sum is taken over all sets $A \subset\{1, \ldots, n\}$ which have exactly $k$ elements.

Due to the factorization of the Hilbert space $\mathcal{H}=\mathcal{H}_{A} \otimes \mathcal{H}_{A^{\prime}}$ where $\mathcal{H}_{A}=\bigotimes_{i \in A} \mathcal{H}_{i}$, $\mathcal{H}_{A^{\prime}}=\bigotimes_{i \notin A} \mathcal{H}_{i}$ we can write each hamiltonian $H_{A}$ as $H_{A}=H_{A}^{0} \otimes \mathbf{1}_{\mathcal{H}_{A^{\prime}}}$ where $H_{A}^{0}$ : $\mathcal{H}_{A} \rightarrow \mathcal{H}_{A}$ is a selfadjoint operator and $\mathbf{1}_{\mathcal{H}_{A^{\prime}}}: \mathcal{H}_{A^{\prime}} \rightarrow \mathcal{H}_{A^{\prime}}$ is the identity. Suppose that each of the particles has $s$ possible states and therefore $\mathcal{H}_{i}=\mathcal{C}^{s}$ and $\mathcal{H}_{A}=\mathcal{C}^{s^{k}}$; we see that $H_{A}^{0}$ can be viewed as a hermitian matrix with $s^{k}$ rows and columns.

Similarly as in the Wigner's model we shall assume that $H_{A}^{0}=\left(a_{i, j}^{A}\right)$ is a hermitian random matrix, i.e. $a_{i, j}^{A}\left(1 \leq i \leq j \leq s^{k}\right)$ are complex Gaussian random variables with the mean 0 and the covariance $E\left(a_{i, j}^{A} a_{k, l}^{A}\right)=E\left(a_{i, j}^{A} \overline{a_{l, k}^{A}}\right)=\frac{1}{\left(\begin{array}{c}n \\ k\end{array}\right) s^{k}} \delta_{i l} \delta_{j k}$. We assume that the entries of different hamiltonians $H_{A}$ are independent.

The above considered random matrices are related to Gaussian unitary ensemble, but it is easy to write a version which is related to Gaussian orthogonal or symplectic ensemble.

1.3. Overview of the article. The goal of this paper is to investigate the limit distribution of the above spectra when $n$ tends to infity and the rank of the interaction $k(n)$ depends in a certain way on $n$.

A classical result of Wigner [Wig1, Wig2] states that if $k(n)=n$ then the distribution of the eigenvalues converges to the semicircular distribution (see Fig. 1). On the other 
hand Mon and French [MF] showed that if $k$ is constant and $n$ converges to infinity then the distribution of eigenvalues converges to the Gaussian distribution (see Fig. 6).

In this article we show that in the intermediate cases when $1 \ll k(n) \ll n$ the limit distributions are given by so-called $q$-deformed Gaussian distributions.

2. The $q$-deformed Gaussian random variables. For overview articles on $q$-deformed commutation relations and $q$-deformed Gaussian variables we refer to [FB, BS1, BS2, BKS, vLM, Sn1, Sn, Spe]. In this article we will consider only one $q$-deformed Gaussian variable, which simplifies the discussion significantly.

Let us consider a real number $-1<q<1$. One says that an operator $a$ acting on a some Hilbert space $\mathcal{K}$ and its adjoint $a^{\star}$ fulfil the $q$-deformed commutation relations if

$$
a a^{\star}-q a^{\star} a=\mathbf{1},
$$

where $\mathbf{1}$ is the identity operator.

Suppose that there exists a unital vector $\Omega \in \mathcal{K}$ such that

$$
a \Omega=0 .
$$

A vector with this property is called a vacuum. In such a setup we can introduce a (non-commutative) expectation value $\tau(X)=\langle\Omega, X \Omega\rangle$.

By the $q$-deformed Gaussian random variable we mean the operator $a+a^{\star}$.

The $q$-deformed Gaussian distribution is a compactly supported probability measure $\nu_{q}$ on the real line with the property that for each natural number $n$ we have

$$
\int x^{n} d \nu_{q}(x)=\tau\left[\left(a+a^{\star}\right)^{n}\right]=\left\langle\Omega,\left(a+a^{\star}\right)^{n} \Omega\right\rangle .
$$

It can be proven [Sz] that for $-1<q<1$ this measure is supported on the interval $[-2 / \sqrt{1-q}, 2 / \sqrt{1-q}]$ and the density of this measure is given by

$$
\nu_{q}(d x)=\frac{1}{\pi} \sqrt{1-q} \sin \theta \prod_{n=1}^{\infty}\left(1-q^{n}\right)\left|1-q^{n} e^{2 i \theta}\right|^{2} d x,
$$

where $\theta \in[0, \pi]$ is defined by $x=\frac{2}{\sqrt{1-q}} \cos \theta$.

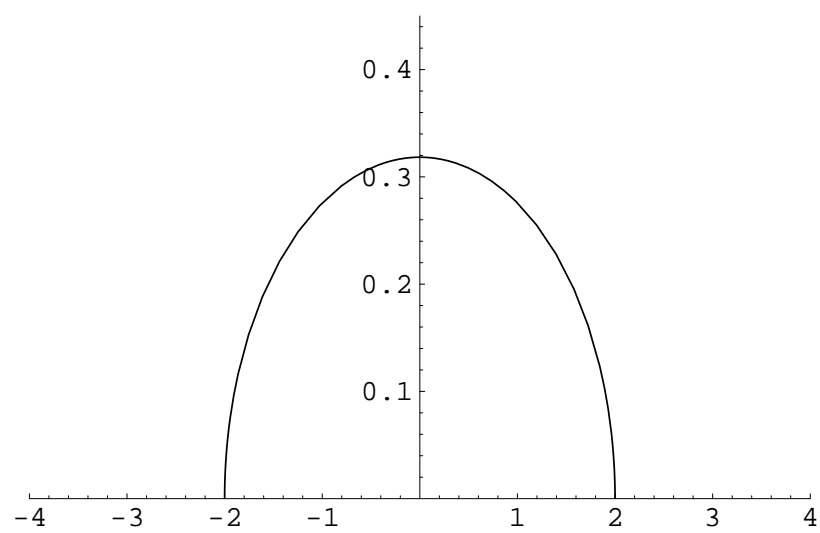

Fig. 1. Wigner semicircular law, corresponding to $q=0$ 


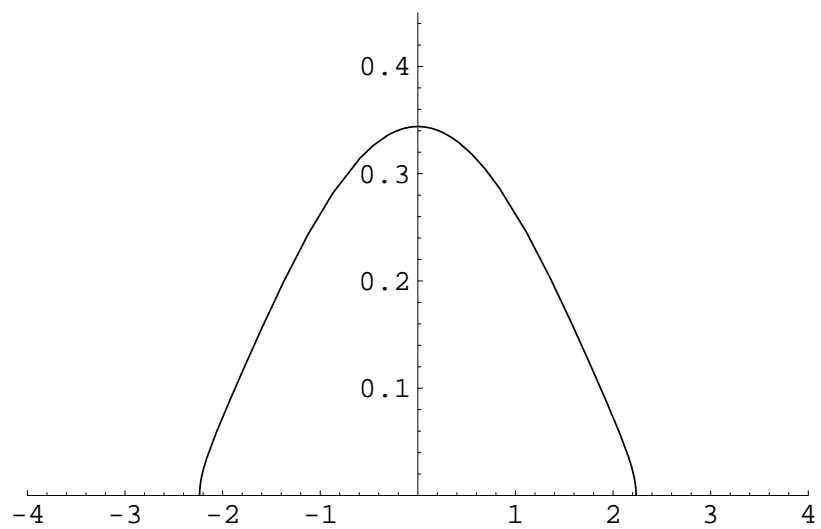

Fig. 2. The $q$-deformed distribution for $q=0.2$

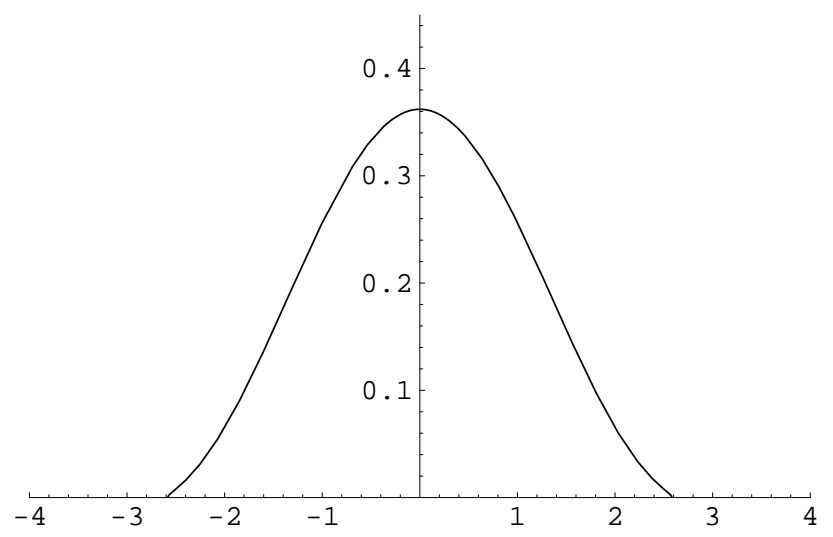

Fig. 3. The $q$-deformed distribution for $q=0.4$

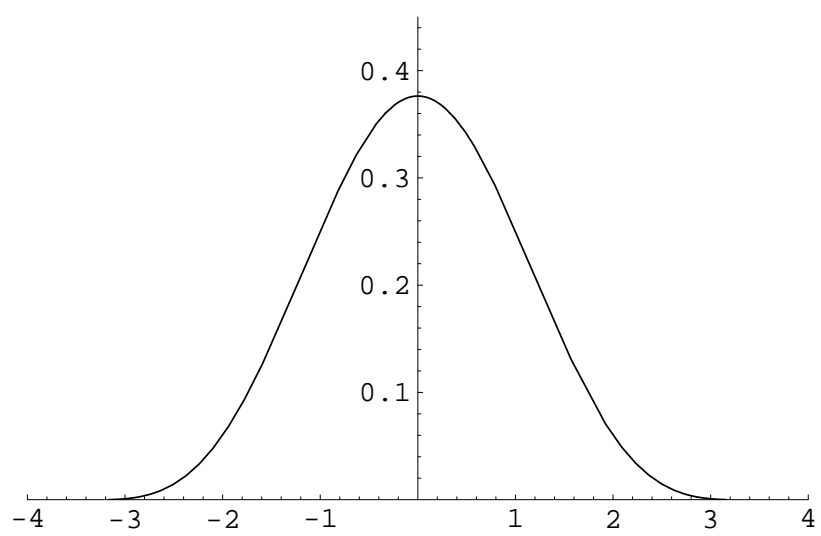

Fig. 4. The $q$-deformed distribution for $q=0.6$ 


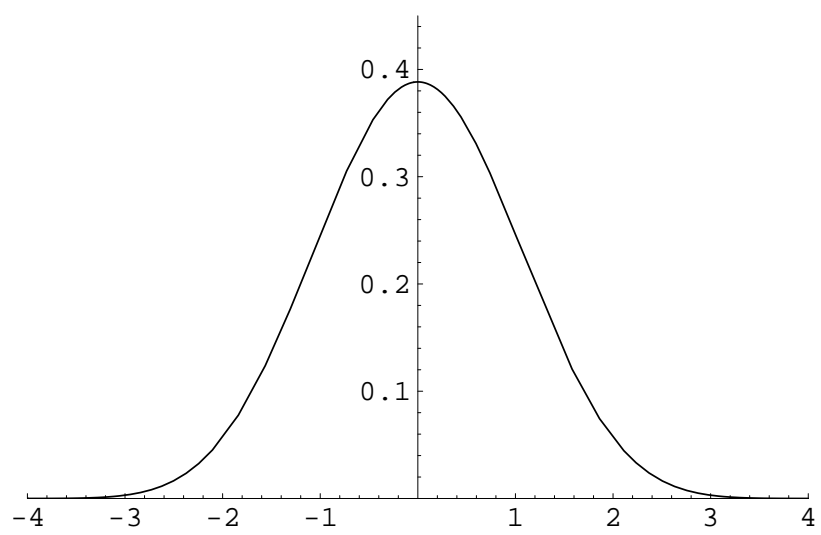

Fig. 5. The $q$-deformed distribution for $q=0.8$

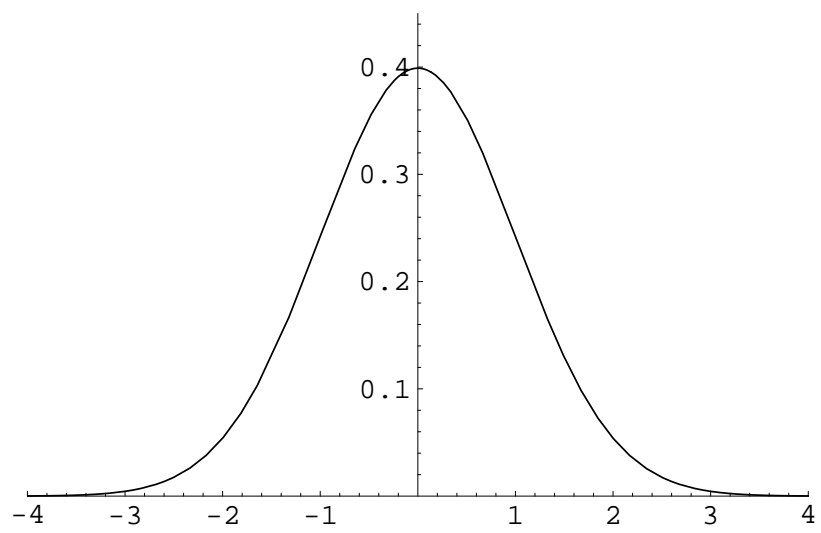

Fig. 6. The Gaussian distribution, corresponding to $q=1$

It turns out that for $q=1$ the $q$-deformed Gaussian distribution coincides with the standard Gaussian distribution (see Fig. 6). For $q=0$ the $q$-deformed Gaussian distribution coincides with the semicircular distribution of Wigner (see Fig. 1). The $q$ deformed Gaussian distributions for intermediate values of the deformation paramater $q$ are presented in Figures 2-5.

3. The distribution of eigenvalues. The connection of the random matrix models considered in Introduction with $q$-deformed Gaussian variables was found by the author in the following theorem [Sn].

ThEOREM 1. If $\lim _{n \rightarrow \infty} k(n) / \sqrt{n}=c$, where $0 \leq c \leq \infty$ then the limit distribution of the eigenvalues of the hamiltonians $H$ is the q-deformed Gaussian distribution with the parameter $q$ given by

$$
q=\exp \left[-\left(1-\frac{1}{s^{2}}\right) c^{2}\right],
$$

where $s$ is the number of one-particle states. 
It should not be a surprise to see that the above theorem contains results of Wigner (in the model considered by him we have $k=n$ and $\lim _{n \rightarrow \infty} k / \sqrt{n}=\infty, q=\exp [-\infty]=0$ ) and of Mon and French ( $k$ is constant, therefore $c=0$ and hence $q=1$ ).

We see that the square root from the number of particles is the scale of the rank of the interaction in which the passage from the Gauss law to the semicircular law occurs.

4. Final remarks. Particles considered in this article were distinguishable. The next step would be to replace them by fermion or bosons. In the fermionic case it would mean for example that $s$, the dimension of a one-particle Hilbert space must be a function of $n$, the number of particles. Simple arguments show that if $s(n)$ tends to infinity faster than linearly, then most of the states are not occupied and the Pauli exclusion principle does not affect our calculations. In this case we can take $s=\infty$ and therefore

$$
q=e^{-c^{2}} \text {. }
$$

Acknowledgements. Research supported by State Committee for Scientific Research (Komitet Badań Naukowych) grant No. 2P03A00723; by EU Network "QP-Applications", contract HPRN-CT-2002-00729; by KBN-DAAD project 36/2003/2004.

\section{References}

[BF1] O. Bohigas and J. Flores, Phys. Letters 34B (1971), 261.

[BF2] O. Bohigas and J. Flores, Phys. Letters 35B (1971), 383.

[BKS] M. Bożejko, B. Kuemmerer and R. Speicher, q-Gaussian processes: Non-commutative and classical aspects, Commun. Math. Phys. 185 (1997), 129-154.

[BS1] M. Bożejko and R. Speicher, An example of a generalized Brownian motion, Commun. Math. Phys. 137 (1991), 519-531.

[BS2] M. Bożejko and R. Speicher, Completely positive maps on Coxeter groups, deformed commutation relations and operator spaces, Math. Ann. 300 (1994), 97-120.

[FB] U. Frisch and R. Bourret, Parastochastics, J. Math. Phys. 11 (1970), 364-390.

[FW1] J. B. French and S. S. M. Wong, Phys. Letters 33B (1970), 449.

[FW2] J. B. French and S. S. M. Wong, Phys. Letters 35B (1971), 5.

[vLM] P. van Leuven and R. Maasen, On q-deformation of the Gauss distribution, J. Math. Phys. 36 (1995), 4743-4756.

[MF] K. K. Mon and J. B. French, Statistical properties of many-particle spectra, Annals of Physics 95 (1975), 90-111.

[Sn1] P. Śniady, On q-deformed quantum stochastic calculus, Probab. Math. Statist. 21 (2001), 231-251.

[Sn] P. Śniady, A Gaussian random matrix model for q-deformed Gaussian variables, Commun. Math. Phys. 216 (2001), 515-537.

[Spe] R. Speicher, A non-commutative central limit theorem, Math. Z. 209 (1992), 55-66.

[Sz] G. Szegö, Ein Beitrag zur Theorie der Thetafunktionen, Sitz. Preuss. Akad. Wiss. Phys. Math. L1 19 (1926), 242-252.

[Wig1] E. P. Wigner, Random matrices in physics, SIAM Review 9 (1967), 1-23.

[Wig2] E. P. Wigner, On the distribution of the roots of certain symmetric matrices, Ann. of Math. 65 (1957), 203-207.

[WF] S. S. M. Wong and J. B. French, Nucl. Phys. A198 (1972), 188. 